\title{
Pour un transfert des savoirs lexicographiques
}

\author{
Alain Polguère \\ OLST - Département de linguistique et de traduction \\ Université de Montréal \\ C.P. 6128, succ. Centre-ville \\ Montréal (Qc) H3C 3J7, Canada \\ alain.polguere@umontreal.ca
}

\section{Introduction}

Les savoirs impliqués dans l'activité lexicographique sont avant tout de nature professionnelle. Il s'agit de connaissances et de compétences théoriques et pratiques, développées au cours des siècles par les lexicographes et que l'on associe presque exclusivement à la tâche de rédaction de dictionnaires ou de bases de données lexicales. Dans ce texte, nous proposons d'envisager les savoirs lexicographiques de façon beaucoup plus générale, en portant plus particulièrement notre attention sur le domaine de l'enseignement et apprentissage de la langue. Nous procéderons en trois étapes. Tout d'abord, nous expliciterons ce que nous entendons par savoirs lexicographiques (connaissances linguistiques impliquées, principes descriptifs et méthodologiques, etc.). Nous détaillerons ensuite les raisons qui nous poussent à envisager l'exploitation de ces savoirs dans le contexte de l'enseignement de la langue. Finalement, nous illustrerons notre propos à partir des travaux que nous avons entrepris pour une exploitation pédagogique des notions et principes de la Lexicologie Explicative et Combinatoire (LEC).

\section{Nature des savoirs lexicographiques}

En explorant la notion de savoirs lexicographiques, nous cherchons à répondre à une question en apparence toute simple : qu'est-ce qu'un lexicographe expérimenté sait que ne sait pas le commun des mortels? Au cours de la présente discussion, nous utilisons le terme pluriel savoirs pour chapeauter la distinction maintenant bien établie entre :

1. les connaissances que l'on possède sur un ou plusieurs objets d'étude donnés, ou savoirs déclaratifs ;

2. les compétences que l'on possède dans la mise en œuvre des tâches de type résolution de problème impliquées par une discipline donnée, ou savoirs procéduraux.

Dans le contexte qui nous intéresse ici, nous devons bien entendu tenter de caractériser la nature même des savoirs lexicographiques ; mais nous devons aussi nous interroger sur la façon dont les connaissances et compétences lexicographiques interagissent dans le processus d'analyse des phénomènes lexicaux. Comme nous le montrerons plus bas, ce processus est, bien entendu, au cœur de l'activité lexicographique, mais il est aussi central dans les activités didactiques liées à l'enseignement et apprentissage des connaissances lexicales - nous dirons, dorénavant, enseignement et apprentissage du vocabulaire.

Nous commençons par récapituler, très brièvement, où en est la lexicographie en tant que discipline (section 2.1). Ensuite, nous défendons l'idée d'une meilleure organisation et formalisation des savoirs lexicographiques (section 2.2). 


\section{Lexicographie et savoirs lexicographiques : un bref état des lieux}

Le terme lexicographie (angl. lexicography) désigne le plus souvent, dans la tradition française et anglosaxonne, la discipline visant l'écriture de dictionnaires. Il s'agit d'un domaine d'activité très ancien, qui a fortement évolué au cours des siècles (Van Hoof, 1994 ; Quemada, 1968), principalement sous l'influence des trois paramètres suivants :

1. la description du lexique de langues très diverses ;

2. l'influence des métaconnaissances sur les langues issues des sciences du langage ;

3. l'évolution des technologies du traitement de l'information.

Ce dernier point fait surtout référence à la généralisation du recours à l'outil informatique dans le travail du lexicographe. Cet outil permet de construire les dictionnaires sous forme de bases de données lexicales - en remplacement des classeurs de fiches lexicographiques (Pruvost, 2000) — et d'utiliser les corpus informatisés comme source de données sur la langue (Stubbs, 2001). Les savoirs du lexicographe ont toujours relevé de ce que l'on pourrait appeler en anglais a craft : la maîtrise d'une discipline se situant entre une technique, un métier et un art (Landau, 1984). Comme pour toute discipline de cette nature, l'acquisition des savoirs lexicographiques se fait par un long et progressif apprentissage, fondé en grande partie sur la pratique de l'activité lexicographique elle-même. Le développement de la linguistique moderne et la généralisation de l'utilisation de l'informatique ont rendu possible l'émergence d'une «nouvelle» lexicographie dans la seconde moitié du $\mathrm{XX}^{\mathrm{e}}$ siècle. Celle-ci possède toutes les caractéristiques fondamentales de la lexicographie traditionnelle qui viennent d'être décrites; elle présente aussi une plus grande structuration du fait des concepts scientifiques, techniques et méthodologiques qu'elle met en jeu.

Différentes approches structurées de la description lexicale ont été proposées (Zgusta, 1971 ; Hartmann, 1983 ; Wierzbicka, 1985 ; Sinclair, 1987 ; Svenson, 1994 ; Mel'čuk et coll., 1995). Les principales équipes lexicographiques adoptent toutes des «théories » lexicologiques et lexicographiques données pour mener à bien leurs tâches descriptives, même si les théories en question peuvent être plus ou moins explicites. Ce qui ressort de toutes les approches modernes du travail lexicographique, c'est l'importance croissante du recours aux nouvelles sources de données sur la langue (corpus informatisés et Internet). Le lexique décrit dans un dictionnaire est, comme la grammaire présentée dans les grammaires usuelles, une entité aux contours flous, une abstraction ou idéalisation d'un code soumis aux variations régionales, sociales, diachroniques, etc. Dans un tel contexte, plus on peut observer de données et plus diversifiées sont les sources de ces données, plus on est en mesure de faire une description ayant une couverture et une précision acceptables. L'étude et le traitement informatique des données lexicales ont donné naissance à une discipline à part entière, à mi-chemin entre la lexicographie, l'étude de corpus et la modélisation des connaissances. Puisqu'elle vise la théorisation des savoirs et techniques d'analyse des phénomènes lexicaux, nous avons appelé cette discipline lexicanalyse (Polguère, 2003a:196). Quel que soit le nom qu'on lui donne, le travail d'accès aux données, de traitement de celles-ci, de préparation des modèles dérivés que sont les bases de données lexicales, est devenu une discipline-clé dans l'étude de la langue (Quemada, 1987).

\section{Pour une modélisation organisée des savoirs lexicographiques}

De nombreux chercheurs et lexicographes ont écrit sur les différents concepts du domaine et ont décrit les techniques de lexicanalyse utilisées dans le cadre de la modélisation lexicale. On doit cependant constater qu'il n'existe pas à l'heure actuelle de modélisation complète et rigoureuse des connaissances et des compétences mises en jeu dans le travail lexicographique. Parmi les connaissances lexicographiques, on peut mentionner les quatre catégories suivantes :

1. concepts linguistiques fondamentaux structurant l'étude du lexique ;

2. concepts propres à la modélisation lexicale dans les dictionnaires et les bases de données ; 
3. concepts liés à l'accès aux données linguistiques ;

4. concepts liés aux tâches descriptives (niveau de description, interdépendance des différents éléments descriptifs, critères et principes descriptifs...).

Les compétences peuvent, quant à elles, être classifiées ainsi :

1. ordonnancement des tâches impliquées dans l'activité de description lexicographique ;

2. interdépendance de ces tâches ;

3. résolution des conflits lorsque plusieurs principes descriptifs ou types de données sont en contradiction.

Bien entendu, un riche bagage a déjà été accumulé sur chacun des points mentionnés ci-dessus, et nombre de publications ont présenté de façon partielle comment tel ou tel aspect du problème de la description lexicale pouvait être modélisé. Ce qui nous manque, c'est un véritable modèle intégré de tous ces savoirs, modèle de type ontologique, qui permettrait de traiter l'activité lexicographique comme un cas particulier de résolution de problème (problem solving).

Dans ce contexte, nous venons d'entreprendre à l'Observatoire de linguistique Sens-Texte (OLST) de l'Université de Montréal une recherche visant l'identification et l'ontologisation des savoirs lexicographiques en vue de leur transfert, notamment, dans le domaine de l'enseignement de la langue. Ce projet, appelé Lexitation, s'appuie en grande partie sur une approche expérimentale de l'identification des savoirs lexicographiques: simulation «en laboratoire» de l'activité lexicographique suivie d'une analyse et modélisation formelle de cette dernière.

\section{Transfert des savoirs lexicographiques}

Nous nous intéressons ici principalement au transfert des savoirs lexicographiques vers le domaine de l'enseignement et apprentissage du vocabulairii. Tout d'abord (section 3.1), nous proposons quelques réflexions sur la place (actuelle et souhaitable) du lexique dans l'enseignement de la langue. Ensuite (section 3.2), nous tentons de convaincre nos lecteurs de la nécessité d'effectuer, dans le contexte du transfert des savoirs lexicographiques vers le domaine de l'enseignement de la langue, une formalisation aussi complète et systématique que possible des savoirs en question.

\section{Place de l'étude du lexique dans l'enseignement de la langue}

Les connaissances lexicales sont au cœur de la connaissance linguistique puisque la langue est un système sémiotique complexe constitué de signes qui sont, dans leur immense majorité, de nature lexicale. Le lexique est une nébuleuse de plusieurs centaines de milliers d'unités, si l'on tient compte de la polysémie des vocables et de la présence des unités lexicales nultilexémiques (locutions). De plus, chaque élément de ce réseau informationnel est lui-même associé à son propre treillis de caractéristiques : sens, formes (écrites et orales), propriétés de combinatoire et réseau de liens sémantiques qui connectent chaque unité lexicale aux autres unités lexicales de la langue. On le voit, la connaissance lexicale est un tout immense, complexe et relativement hétérogène. Il ne s'agit pas d'une simple collection de mots, mais bien d'un système lexical (Polguère, 2006). On pourrait alors s'étonner, comme le fait Picoche (1984), que si peu d'efforts aient été investis depuis que la scolarisation existe dans l'enseignement des notions générales permettant de comprendre l'organisation du lexique. Cet état de fait peut cependant s'expliquer. Tout d'abord, l'enseignement linguistique au primaire et au secondaire a traditionnellement été centré, non sur le développement d'une capacité à apprendre des notions et techniques permettant de décrire la langue, mais sur la mémorisation de règles normatives (Wilmet, 2000). Ainsi, l'enseignement classique de la grammaire consiste à faire apprendre des règles de grammaire, et non à montrer comment on peut mettre au jour par soi-même de telles règles à partir de l'observation des faits grammaticaux. Même si l'on assiste à l'émergence de nouvelles stratégies dites « actives » pour compenser les manques de l'approche traditionnelle (Chartrand, 1995), on est encore loin de voir une généralisation de ce type de méthodes. 
Dans le cas du lexique, comme il n'est pas envisageable de faire mémoriser aux apprenants des articles de dictionnaires, on se limite souvent à la mémorisation de « listes de vocabulaire » (au primaire) en espérant que le reste de l'acquisition des connaissances lexicales se fera par simple imprégnation. Ce faisant, aussi bien les élèves que les enseignants sont rarement entraînés à décrire les phénomènes lexicaux et à les comprendre. Un résultat typique de ce manque de préparation à la compréhension du lexique est que nombre de problèmes rencontrés par les apprenants sont mal identifiés et, donc, mal traités. Parce qu'il est impossible d'isoler un ensemble simple et clairement délimité de "règles lexicales » qu'il faudrait enseigner, on ignore souvent le lexique comme composante centrale de la connaissance linguistique et on passe à côté de la véritable solution : il faut apprendre à observer et à décrire les phénomènes lexicaux pour mieux acquérir la connaissance lexicale. Il ne s'agit pas tant ici d'apprendre à « utiliser des dictionnaires »(Lipp, 1992) que d'acquérir les notions fondamentales qui structurent la connaissance lexicale et sa modélisation — voir à ce propos Simard (1994).

\section{Nécessité d'un transfert vers le domaine pédagogique}

Nous avons la conviction qu'il faut sortir la lexicographie de son champ d'application traditionnel (la construction de dictionnaires ou bases de données lexicales) pour lui donner un statut de champ de compétences général. Dans ce contexte, nous voyons l'élaboration d'un modèle complet et bien formalisé des savoirs lexicographiques, du type de celui mentionné plus haut (section 2.2) à propos du projet Lexitation, comme un outil essentiel de propagation de ces savoirs vers le domaine didactique.

Un modèle relativement complet et formalisé des savoirs lexicographiques aurait une influence directe sur l'écriture de descriptions lexicales. Compte tenu des enjeux associés à la construction de grandes bases de données lexicales en traitement automatique des langues (Wilks et coll., 1996 ; Fellbaum, 1998), on peut considérer que cette application directe d'un modèle lexicographique justifie en soi que l'on s'y intéresse. Cependant, il existe d'autres champs d'application de ces savoirs que la seule lexicographie ; nous faisons notamment le constat suivant :

la maîtrise des savoirs lexicographiques n'est pas seulement nécessaire dans les tâches propres à la construction de dictionnaires et de bases de données lexicales : elle renforce considérablement les compétences professionnelles de toute personne impliquée dans l'enseignement de la langue.

Prenons le cas particulier des enseignants de français du primaire. Dans leur interaction avec les élèves, ils sont constamment amenés à répondre à des questions sur les mots de la langue, à établir des diagnostics sur des erreurs de nature lexicale et à aider les élèves à trouver des solutions à leurs problèmes d'expression. Dans la plupart des cas, ces enseignants, qui n'ont pas appris à déduire des modèles de la langue à partir de leurs propres observations, cherchent à donner des réponses fondées sur ce qu'ils pensent être inscrit dans les dictionnaires. Même s'il est impensable, pour des raisons pédagogiques et pratiques, que les enseignants aillent à chaque fois consulter un dictionnaire (ou une grammaire) avant d'émettre un avis sur la langue, ils voudraient sans doute la plupart du temps pouvoir le faire. Pourquoi ? Parce que la connaissance linguistique, notamment lexicale, est souvent perçue comme un savoir statique, normalisé, que l'on ne peut modéliser de façon simple et rapide au moyen d'un ensemble de techniques aisément accessibles. Modéliser le lexique serait strictement une affaire de lexicographes professionnels. Ceux-ci auraient pour fonction sociale de rendre disponibles des modèles de référence, comme si la construction ou, du moins, l'ébauche de tels modèles n'était pas envisageable en dehors de la lexicographie professionnelle.

Nous ne remettons nullement en question le fait que la lexicographie soit une affaire de spécialistes. Bien au contraire, nous savons d'expérience à quel point elle est fondée sur des savoirs difficiles à acquérir. Cependant, l'observation que nous avons pu faire de l'apprentissage de la lexicologie par les étudiants (dont un grand nombre se destinent à l'enseignement des langues) nous a convaincu que les savoirs lexicographiques augmentent considérablement l'autonomie disciplinaire du professionnel de la langue et lui permettent de fonctionner de façon optimale dans les situations où l'analyse des faits linguistiques doit se faire instantanément, comme dans les contextes d'enseignement. Si l'on reconnaît l'importance 
primordiale de la bonne maîtrise de langue dans la vie de nos sociétés, les quelques remarques qui viennent d'être faites ne peuvent que nous convaincre de la nécessité, et non simplement de l'utilité pratique, d'un transfert des savoirs lexicographiques vers le domaine de l'enseignement.

On trouvera dans Polguère et Tremblay (2003) plusieurs propositions concrètes sur la façon dont les savoirs lexicographiques peuvent être mis en pratique dans l'enseignement des connaissances lexicales. Tel que l'illustre la citation ci-dessous, l'accent est mis par les auteurs sur la nécessité de structurer la démarche pédagogique en fonction de l'organisation même des phénomènes linguistiques qu'il s'agit de prendre en charge :

Nous postulons qu'il importe avant tout d'explorer deux types de phénomènes, qui se manifestent à travers l'ensemble du lexique, y compris dans le cas des mots tout à fait courants de la langue. Il s'agit de

- la polysémie, qui doit nous forcer à isoler les différentes acceptions d'un mot pour pouvoir les caractériser et les différencier ;

- la connexion lexicale, qui fait qu'une unité lexicale n'existe que par (i) son positionnement dans le réseau lexical de la langue (liens paradigmatiques) et (ii) ses propriétés individuelles, qui contrôlent la façon dont elle se combine aux autres unités lexicales dans la phrase (liens syntagmatiques).

Bien entendu, un transfert des savoirs lexicographiques vers l'enseignement de la langue consiste non seulement à inculquer aux enseignants de nouvelles connaissances et compétences, mais aussi à leur fournir des outils descriptifs sur lesquels ils peuvent s'appuyer pour mettre en œuvres des stratégies d'enseignement du vocabulaire; il s'agit avant tout des dictionnaires ou bases lexicales spécialement conçus pour appuyer l'étude des phénomènes identifiés dans la citation ci-dessus (structure polysémique des vocables et connexions paradigmatiques ou syntagmatiques entre unités lexicales). On mentionnera ici notamment, pour le français, le Dictionnaire du français usuel (Picoche, 2002) et le Lexique actif du français (Mel'čuk et Polguère, 2007). Nous aurons l'occasion de revenir sur ce dernier ouvrage dans la section qui suit.

\section{Exploitations pédagogiques de la Lexicologie Explicative et Combinatoire}

Dans cette dernière section, nous allons donner quelques informations sur l'approche que nous adoptons pour tenter de faire progresser le transfert des savoirs lexicographiques que nous prônons ici. Nous commencerons (section 4.1) par brièvement justifier l'intérêt du cadre théorique et descriptif dans lequel nous nous situons: la Lexicologie Explicative et Combinatoire. Nous dirons ensuite quelques mots (section 4.2) sur deux ressources spécifiques qui ont été développées selon cette approche afin de répondre, en tout premier lieu, aux besoins des enseignants du français.

\section{Intérêt de la Lexicologie Explicative et Combinatoire}

La lexicographie moderne est intimement liée à l'étude scientifique des lexiques des langues naturelles, c'est-à-dire à la lexicologie. Il est clair qu'un travail de modélisation et de transfert des savoirs lexicographiques doit s'appuyer sur un cadre théorique bien défini, afin d'assurer la cohérence conceptuelle du modèle à construire. Pour ce qui est du cadre lexicologique de référence, nous nous appuyons dans nos travaux sur la Lexicologie Explicative et Combinatoire ou LEC, qui est la branche lexicale de la théorie linguistique Sens-Texte. La présentation qui en est faite dans la littérature linguistique (Mel'čuk et coll., 1995; Polguère, 2003a) correspond à ce que l'on pourrait appeler une ontologie semi-formelle de cette théorie du lexique : réseau notionnel et ensemble de critères et de méthodes présentés en langue naturelle, mais en suivant des contraintes d'expression et des contraintes terminologiques strictes.

L'approche de la LEC est, selon nous, particulièrement appropriée pour servir au développement d'une modélisation formelle et à un transfert des savoirs lexicographiques pour les raisons suivantes : 
1 elle est formelle (notions descriptives et formalismes de représentation précisément définis) et possède une valeur « universelle », ayant été appliquée à une grande variété de langues très diverses ;

2 elle vise spécifiquement la production de modèles lexicaux, appelés Dictionnaires explicatifs et combinatoires ou DEC (Mel'čuk et coll., 1984, 1988, 1992, 1999), et est appliquée à la construction de bases de données lexicales formelles, manipulables informatiquement - cf. la base de données DiCo du français (Polguère, 2000a; Mel'čuk et Polguère, 2006) ou la base bilingue de collocations de Fontenelle (1997);

3 elle sert aussi à la construction de modèles « vulgarisés » adaptés à l'enseignement et apprentissage de la langue - cf. le Lexique Actif du Français ou LAF (Polguère, 2000b ; Mel'čuk et Polguère, 2007) ou le dictionnaire en ligne DAFLES (Selva et coll., 2003);

4 son potentiel d'application à la didactique des langues — déjà examiné dans le passé (Leed et Nakhimovsky, 1990 ; Steele, 1990 ; Gentilhomme, 1992) - a été confirmé par des études récentes (Tremblay, 2003) et intéresse de plus en plus les chercheurs en didactique; elle est de plus utilisée avec succès dans d'autres domaines de linguistique appliquée, comme la terminologie (L'Homme, 2002).

L'énumération ci-dessus n'a pas pour finalité de démontrer que la LEC est la seule et unique approche disponible «sur le marché » pour mettre en œuvre le transfert des savoirs lexicographiques. Elle vise simplement à justifier le caractère raisonnable de son utilisation pour atteindre les buts que nous nous fixons.

\section{Efforts de transfert entrepris dans le cadre de la LEC}

Le projet Lexitation de modélisation formelle des connaissances lexicographiques ne faisant que débuter (voir section 2.2), les seuls efforts de transfert des notions de la LEC que nous allons mentionner ici sont ceux visant la production de ressources lexicographiques adaptées à l'enseignement et apprentissage des connaissances lexicales : il s'agit du Lexique actif du français ou LAF et du DiCoPop.

\subsection{Le LAF : un manuel de lexicologie fondé sur un échantillon de dictionnaire}

Le LAF, rédigé en collaboration avec Igor Mel'čuk, est un travail qui a déjà mentionné à la section 4.1. En tant qu'ouvrage publié, il tire son originalité du fait qu'il est à la fois un manuel de lexicologie destiné, en tout premier lieu, aux enseignants de langue et un échantillon de dictionnaire du français, reposant sur une adaptation des descriptions formalisées de la LEC. Il s'accompagne d'un site web, où sont notamment rendus disponibles pour les enseignants de français des modèles d'exercices visant l'apprentissage du vocabulairiii. Par sa finalité et par sa double nature (présentation de notions lexicologiques et de descriptions lexicographiques), le LAF peut être rapproché de Picoche (2007). Il est intéressant de constater que le travail d'interfaçage des principes et descriptions de la LEC opéré lors de la rédaction du LAF a permis, de façon rétroactive, de faire progresser l'approche théorique elle-même. On trouvera un bilan de l'expérience acquise au cours de la rédaction du LAF dans Polguère (2007). Dans ce texte, on fait notamment état des innovations introduites pour ce qui est de la caractérisation sémantique des unités lexicales (au moyen d'étiquettes sémantiques) et de l'encodage des relations lexicales paradigmatiques et syntagmatiques (au moyen de formules dites « de vulgarisation »).

Une autre caractéristique originale du LAF est sa méthodologie d'élaboration (Polguère, 2000b). Il est en effet entièrement dérivé de la base lexicale DiCo des dérivations sémantiques et collocations du français, développée par Igor Mel'čuk et le présent auteur. Cette façon de procéder assure au LAF une rigueur formelle sous-jacente et, surtout, nous permet de dériver de la base source DiCo d'autres "produits », comme celui dont il va maintenant être question. 


\subsection{Le DiCoPop : un LAF virtuel et bien plus}

Le DiCoPop est une première étape vers la réalisation de ce que Jousse et coll. (2008) appellent un site lexical. Il équivaut pour l'instant à un dictionnaire en ligne, très proche du LAF, et qui est tout comme ce dernier entièrement dérivé de la base $\mathrm{DiCo}^{\mathrm{iv}}$. Outre le fait qu'il ne s'agit pas d'un ouvrage papier, deux caractéristiques importantes distinguent le DiCoPop du LAF. Tout d'abord, les descriptions du DiCoPop sont extraites du DiCo de façon entièrement automatique, sans intervention des lexicographes pour ce qui est de la «pédagogisation » des descriptions lexicales de la base source. Ensuite, et cela est fondamental, le DiCoPop n'existe, en tant que dictionnaire, que de façon virtuelle. Les descriptions lexicographiques qu'obtiennent les utilisateurs du DiCoPop sont construites à la volée, en réponse aux requêtes particulières adressées à la base. Le DiCoPop est donc bien un dictionnaire virtuel, au sens de Atkins (1996). Cette caractéristique du DiCoPop offre les trois avantages suivants :

1. il est constamment à jour, puisque l'information qu'il procure est toujours extraite en temps réel du contenu courant de la base DiCo ;

2. il a le potentiel d'offrir de multiples types de navigations dans les données lexicales, notamment des navigations fondées sur le (i) la hiérarchie des étiquettes sémantiques du DiCo et (ii) le réseau de liens paradigmatiques et syntagmatiques tissé par le système des fonctions lexicales standard de la théorie Sens-Texte (Mel'čuk, 1996);

3. il permet d'obtenir descriptions qui ne gravitent pas nécessairement autour d'une unité lexicale donnée (articles de dictionnaire) : on peut notamment obtenir de l'information sur des classes sémantiques (grâce aux étiquettes sémantiques).

\section{Conclusion}

Dans ce qui précède, nous avons brièvement tenté de présenter et défendre les trois idées suivantes :

1. les savoirs lexicographiques - mis en jeu dans l'activité de rédaction de dictionnaires et bases de données lexicales - peuvent et doivent être modélisés de façon systématique et formelle en vue d'une meilleure compréhension de leur organisation et d'une meilleure exploitation ;

2. ces savoirs doivent être transférés et devenir, au moins partiellement, une composante du champ de compétences générales que chacun possède sur la langue ;

3. le transfert de ces savoirs est une nécessité dans le domaine de la didactique de la langue, pour ce qui est de l'enseignement et apprentissage du vocabulaire.

Plutôt que d'offrir un panorama général de ce qui se fait en lexicologie et lexicographie dans le cadre du transfert des savoirs lexicographiques, nous avons préféré nous concentrer sur l'explicitation de nos propres idées et des efforts que nous avons entrepris en ce sens. En effet, il nous semble, peut-être à tort, que l'idée même de la nécessité d'un transfert des savoirs lexicographiques vers le domaine de l'enseignement et apprentissage du vocabulaire est très peu répandue. Selon ce que nous savons, elle est essentiellement mise en pratique en didactique des langues dans le cadre d'exercices ponctuels de rédaction d'articles ou de parties d'articles de dictionnaire (définition, etc.) qu'élaborent les enseignants de langue. Ajoutons que, généralement, ces exercices sont conçus sans qu'il soit fait appel à une modélisation suffisamment complète des savoirs lexicographiques. Nous serions personnellement très heureux de recevoir toute information sur des projets et des initiatives pédagogiques allant dans le même sens que les idées que nous défendons ici.

\section{Remerciements}

Les travaux de l'Observatoire de linguistique Sens-Texte (OLST) de l'Université de Montréal mentionnés ici sont financés par le Fonds québécois de recherche sur la société et la culture (FQRSC) et par le Conseil de recherches en sciences humaines du Canada (CRSH). 


\section{Références bibliographiques}

Atkins, B. T. S. (1996) Bilingual Dictionaries: Past, Present and Future. In M. Gellerstam, J. Järborg, S.-G. Malmgren, K. Norén, L. Rogström et C. Röjder Papmehl (dir.) : Proceedings of Euralex'96, Gothenburg, 515546.

Chartrand, S.-G. (dir.) (1995) Pour un nouvel enseignement de la grammaire : propositions didactiques. Collection "Théories et pratiques dans l'enseignement », Montréal : Éditions Logiques.

Fellbaum, C. (dir.) (1998) WordNet: An Electronic Lexical Database. Cambridge, Mass. : The MIT Press.

Fontenelle, T. (1997) Turning a bilingual dictionary into a lexical-semantic database. Tübingen : Niemeyer.

Gentilhomme, Y. (1992) Panorama sur le Dictionnaire Explicatif et Combinatoire: retombées pédagogiques. In Mel'čuk et coll. (1992), DEC III, Montréal : Presses de l’Université de Montréal, 95-120.

Hartmann, R. R. K. (dir.) (1983) Lexicography: Principles and Practice. Londres : Academic Press.

Jousse, A.-L., Polguère, A., Tremblay, O. (2008) Du dictionnaire au site lexical pour l'enseignement/apprentissage du vocabulaire. In F. Grossmann et S. Plane : Lexique et production verbale. Vers une meilleure intégration des apprentissages lexicaux, Collection «Éducation et didactiques », Villeneuve d'Ascq : Presses Universitaires du Septentrion, 141-157.

L'Homme, M.-C. (2002) Fonctions lexicales pour représenter les relations sémantiques entre termes. Traitement automatique de la langue (T.A.L.), 43(1), 19-41.

Landau, S. (1984) Dictionaries: The Art and Craft of Lexicography. New York : Charles Scribner's Sons.

Leed, R., Nakhimovsky, A. (1990) Lexical Functions and Language Learning. In J. Steele (dir.) : Meaning-Text Theory: Linguistics, Lexicography, and Implications, Ottawa/London/Paris : University of Ottawa Press, 365374.

Lipp, B. (1992) L'enseignement du vocabulaire, quels dictionnaires pour quelles activités ? Enjeux, 26, 29-45.

Mel'čuk, I. (1996) Lexical Functions: A Tool for the Description of Lexical Relations in the Lexicon. In L. Wanner (dir.): Lexical Functions in Lexicography and Natural Language Processing, Amsterdam/Philadelphie: Benjamins, 37-102.

Mel'čuk, I., Clas, A., Polguère, A. (1995) Introduction à la lexicologie explicative et combinatoire. Louvain-laNeuve: Duculot.

Mel'čuk, I., Polguère, A. (2006) Dérivations sémantiques et collocations dans le DiCo/LAF. Langue française, 150, numéro spécial sur la collocation «Collocations, corpus, dictionnaires », sous la direction de P. Blumenthal et F. J. Hausmann, 66-83.

Mel'čuk, I., Polguère, A. (2007) Lexique actif du français. L'apprentissage du vocabulaire fondé sur 20000 dérivations sémantiques et collocations du français. Collection «Champs linguistiques », Bruxelles : De Boeck \& Larcier.

Mel'čuk, I. et coll. (1984, 1988, 1992, 1999) Dictionnaire explicatif et combinatoire du français contemporain. Recherches lexico-sémantiques (Volumes I à IV). Montréal : Presses de l'Université de Montréal.

Picoche, J. (1984) Comment enseigner le vocabulaire. L'information grammaticale, 21, 3-7.

Picoche, J. (2007) Enseigner le vocabulaire, la théorie et la pratique. Champigny-sur-Marne : Allouche. [Réédition revue et corrigée, sous forme de CD-ROM, de Didactique du vocabulaire français paru chez Nathan en 1993.]

Picoche J., Rolland J.-C. (2002) Dictionnaire du français usuel, Bruxelles : De Boeck-Duculot.

Polguère, A. (2000a) Une base de données lexicales du français et ses applications possibles en didactique. Revue de Linguistique et de Didactique des Langues (LIDIL), 21, 75-97.

Polguère, A. (2000b) Towards a Theoretically-Motivated General Public Dictionary of Semantic Derivations and Collocations for French. Proceedings of EURALEX'2000, Stuttgart, 517-527.

Polguère, A. (2003a) $)^{v}$ Lexicologie et sémantique lexicale. Notions fondamentales. Collection «Paramètres ", Montréal : Presses de l’Université de Montréal. 
ISBN 978-2-7598-0358-3, Paris, 2008, Institut de Linguistique Française

$$
\text { Lexique(s) }
$$

DOI $10.1051 / \mathrm{cmlf0833}$

i Le projet Lexitation est financé par le Conseil de Recherches en Sciences Humaines (CRSH) du Canada. Autres chercheurs impliqués : Jacqueline Bourdeau, Pascale Lefrançois, Igor Mel'čuk et Claude Rigault. On trouvera plus d'informations sur le projet sur sa page web : $\underline{\mathrm{http}: / / \text { olst.ling.umontreal.ca/recherche/linguistique/lexitation. }}$

ii Cette section reprend certaines des idées initialement présentées dans Polguère (2004).

iii À l'adresse : http://olst.ling.umontreal.ca/laf.

iv L'adresse web du DiCoPop est : http://olst.ling.umontreal.ca/dicopop. Il s'agit d'un projet mené par le présent auteur en collaboration avec Sébastien Cabot, qui a assuré tout le travail de programmation.

Ouvrage maintenant épuisé. Une édition revue et corrigée doit paraître en 2008. 